\title{
Follow-up in melanoma patients
}

\author{
Piotr Rutkowski · Iwona Ługowska
}

Received: 2 February 2014 / Accepted: 10 June 2014 / Published online: 24 June 2014

(C) The Author(s) 2014. This article is published with open access at Springerlink.com

\begin{abstract}
Due to lack of evidence from prospective clinical trials, the diagnostic procedures, their frequency, as well as the length of the follow-up period in cutaneous melanoma patients should be based on the individual risk of disease recurrence, which is strongly dependent on the stage of disease at the time of diagnosis. In the paper we propose the current recommendations for follow-up strategy. Nowadays, new effective treatment options with biological agents justify the closer monitoring of high risk melanoma patients.
\end{abstract}

Keywords Melanoma Follow-up · Recurrences

Due to the absence of clear evidence from prospective clinical trials, the recommendations for melanoma follow-up are mainly based on the retrospective analyses of prognostic factors (e.g., stage of melanoma) and the onset of disease recurrence [1-5]. Other factors, such as the presence of dysplastic nevi, family history, and patient or physician concerns will impact follow-up schedule as well.

The types of diagnostic procedures, their frequency, as well as the length of follow-up period should be based on the individual risk of disease recurrence [5-10]. The risk of recurrence and survival is strongly dependent on the stage of disease at the time of diagnosis and is significantly higher after surgical resection of metastases to regional lymph node than in T1-T2 patients with negative sentinel lymph node [11-15]. Of note, the 5-year recur-

P. Rutkowski , MD, PhD ( $\varangle) \cdot$ I. Ługowska

Department of Soft Tissue/Bone Sarcoma and Melanoma, Maria Sklodowska-Curie Memorial Cancer Center and Institute of

Oncology, Roentgena 5,

02-781 Warsaw, Poland

e-mail: rutkowskip@coi.waw.pl rence free survival in IIIA, IIIB, IIIC stages (according to American Joint Committee on Cancer-AJCC) is equal to $50-63 \%, 26-32 \%$ and $11-12 \%$ as compared with 5 -year recurrence free survival in stages IA, IB, and IIA equal to $95 \%, 82 \%, 72 \%$, respectively $[2,6,13,15,16]$. Because the recurrence rate is the highest in the first 2-3 years after treatment, follow-up visits must be intensified in this period $[17,18]$.

The main goal of the follow-up is to detect a locoregional relapse possible to be treated surgically $[1,9,12$, $13,16,17,19,20]$. The most common site of the first relapse is local relapse or in-transit metastases $(20-28 \%$ of patients), more than $25 \%$ of patients have regional lymph node involvement and $15-50 \%$ of cases present distant metastases. The percentage of relapse to regional lymph node has been reduced after the introduction of sentinel node biopsy as a standard of care. What is important, a large portion of local and locoregional recurrence can be detected by the patient alone (more than $60 \%$ of cases). Therefore, the patients' education in self-control is obligatory, especially the careful assessment of the regional lymph basin and the scar after primary site excision must be performed every 3-6 months. After 5 years of observation, in patients with successfully treated stage I-III melanoma, the probability of recurrence is less than $5 \%[9,20]$. However, it may occur even many years after the diagnosis with equal distribution between locoregional recurrence and disease dissemination.

In oncological centers, the control visits for asymptomatic patients are routinely performed every 3 months during the first 2 years of follow-up, then every 6 months for the next 3 years and once a year-after 5 years of observation. Patients with melanoma in-situ do not require so long-term observations, except for patients with the presence of multiple atypical skin nevi or other risk factors. Due to low risk of recurrence, the frequency of control visits also should be limited to 1-2 visits per year (every 6-12 months) in melanoma stage IA [2, 6, 21]. 
Table 1 Follow-up recommendation in melanoma patients according to American Joint Committee on Cancer (AJCC) staging group

Early stage melanoma (stage IA(-IB) according to AJCC) - after primary site resection.

Locally advanced melanoma no regional metastases detected [stage (IB) IIA-IIC]—after primary site resection and SNB.

Locally advanced melanoma with metastatic nodes, or matted nodes, or in transit met(s)/ satellite(s) (stage IIIA-IIIC)—after primary site resection with lymphadenectomy. Resected local recurrence or nodal metastases from unknown primary site.

Metastatic disease (stage IV)—after treatment.

\section{Recommendation}

In resected low-risk melanomas (pT1A-B), there is no indication to perform any additional tests except history and physical examination (H\&P).

H\&P with attention to complete skin exam, as well as draining lymph nodes, locoregional area, and scar after primary site resection.

Routine imaging/lab tests not recommended.

Ultrasound of regional lymph nodes only in case of $\mathrm{pT} 1 \mathrm{~b}$ melanoma without sentinel lymph node procedure.

Specific signs or symptoms are indications for additional radiologic imaging.

Chest X-ray-optional.

Patients education towards skin self-examination mandatory.

Stage IB may be grouped with stage II patients due to higher risk of recurrence.

H\&P with attention to complete skin exam, as well as draining lymph nodes, locoregional area, and scar after primary site resection.

During the first three years, in IIB-IIC melanoma, CT and/or ultrasound every 6-12 months and annual brain MRI can be considered to screen for recurrent or metastatic disease at the discretion of the physician.

Chest X-ray-optional.

CBC (Complete Blood Count), Liver Function Tests (LFT) and LDH level—optional.

Patients education towards skin self-examination mandatory.

Stage IIC may follow stage III recommendations due to higher risk of recurrence than stage IIIA.

H\&P with attention to complete skin exam, as well as draining lymph nodes, locoregional area, and scar after primary site resection.

USG of the region after the lymphadenectomy.

Chest X-ray.

During the first 3 years, CT and/or ultrasound every 6-12 months and annual brain MRI should be considered to screen for recurrent or metastatic disease.

CBC, LFT, and LDH level.

Patients education towards skin self-examination and alarming symptoms of recurrence - mandatory.

Metastatic lesions assessment (CT, USG, and/or PET/CT).

CBC, LFT, and LDH level.

Patients education towards skin self-examination and alarming symptoms of recurrence-mandatory.

\section{Time-line}

Every 6-12 months for 5 years; and annually thereafter, as clinically indicated.

Every 3-6 months for 2-3 years; then every $6-12$ months for 3 years; and annually thereafter, as clinically indicated.

Every 3 months for 2 years; then every 3-6 months for 3 years; and annually thereafter, as clinically indicated.

An individual plan of follow-up visits.

PET positron emission tomography, CT computed tomography, LDH lactate dehydrogenase, USG ultrasound sonography

In this group of patients, there is no indication to perform any additional tests except history and physical examination (H\&P) and self-examination and the less intensive control schemes have no negative impact on survival [6, 21-23].

During the follow-up visit, it is obligatory to exclude the locoregional recurrence and disease dissemination. The careful assessment of the scar and the regional lymph nodes/lymphatic inflow (spread in-transit) must be performed; ultrasound examination can be utilized. Ultrasound assessment of lymphatic basin has high sensitivity and specificity [24-26]. It is especially useful in cases not undergoing sentinel node biopsy, because the nodal recurrences in the lymphatic basin after negative sentinel node biopsy usually do not exceed $5 \%$ [27]. Moreover, it has been shown that sentinel lymph node dissection reduces subsequent regional lymph node metastases [28, 29].

For the detection of lung metastasis the specificity of chest X-ray is only about $50 \%$, therefore, this examination is of little value in asymptomatic patients with skin melanoma in stage I-II. It has been suggested, that approximately $68 \%$ of recurrences are detected on the basis of clinical symptoms, $26 \%$ due to physical examination (often by the patients who found the subcutaneous tumor), and only $6 \%$ due to chest X-ray. Moreover, data show that there are no positron emission tomography (PET) tests in order to control patients' disease recurrence after treatment of primary cutaneous melanoma.

In patients with detected locoregional recurrencestage III (without any other findings in H\&P, lactated dehydrogenase serum tests, or chest X-ray), the computed tomography (CT) of chest, abdomen, and pelvis may initially detect distant disease dissemination in only $7 \%$ of patients [30,31]. However, during follow-up in stage III patients the asymptomatic relapse can be revealed with CT scans in $72 \%$ [16], which justifies the recommendation of using chest and abdomen CT imaging for early detection of disease recurrence in this group of patients [17]. In this group of patients (with exception of IIIC stage), the role of the brain CT or magnetic resonance imaging (MRI) is not clear, especially taking into account the cost-effectiveness ratio. In stage IIIC melanoma patients Romano et al. found the risk of recurrence in the form of brain metastases in more than $5 \%$ during the first 13 months after locoregional therapy; 
this may justify performing control MRI in this subset of patients [16]. MRI has been proven to be more sensitive and specific in the detection of cerebral metastases than CT or PET-CT [17]. Nowadays, increasing number of guidelines suggest that in stages IIC-IIIC, abdominal and chest radiological imaging (e.g., CT or MRI scan) should be recommended in asymptomatic patients during the first 2-3 years due to higher risk of relapse and for earlier qualifying low-tumor burden patients with disease dissemination to systemic therapy with new effective drugs (BRAF/MEK inhibitors or immunotherapy) [6, 17, 32]. Before the era of BRAF/MEK inhibitors, the intensive radiological tests following earlier treatment have shown limited benefit of survival (about 2 months of increasing of median survival) $[6,17]$.

Clinical symptoms indicating the presence of distant metastases (e.g., liver enzyme abnormalities, bone pain, neurological symptoms, cough, and weakness) are a clear indication to perform dedicated imaging tests, such as: PET-CT, CT, MRI, or bone scan [9, 17].

The monitoring of serum markers such as the liver function tests and serum lactate dehydrogenase (LDH) level might be indicated in selected cases. Although the increased level of serum markers might be the first symptom of metastatic disease (stages III or IV), it does not support enough proof for the initiation of systemic therapy based on test results only. These serum tests are not expensive and widely available. During the followup, there is no need to asses any other biological markers, although data suggests that monitoring of the serum level of S100 B protein may have prognostic utility, especially for patients with locally advanced melanoma [7, 17]. However, further clinical trials are required to confirm these data.

Melanoma patients are also exposed to an increased risk of developing other skin cancers and secondary melanoma. They should be informed about a higher risk of melanoma in their relatives. However, there are no indications for genetic testing. Patients with diagnosis of skin melanoma have a statistically higher risk of developing secondary melanoma in their lifespan ( $10 \%$ of patients will have secondary melanoma). Therefore, it is obligatory to regularly perform dermoscopic skin examinations in this group of patients. In patients with dysplastic nevi syndrome, the entire skin may be assessed with regular photography of the lesions or videodermatoscopy.

In the absence of evidence-based clinical guidance of follow-up it is also important to take into account the patient's individual preferences. Nowadays, new effective treatment options with biological agents justify the closer monitoring of high risk melanoma patients. The proposed recommendation for follow-up strategy of cutaneous melanoma patients is presented in Table 1 . The radiological detection of asymptomatic metastases may allow for an earlier therapy initiation, which may have favorable influence on prognosis and the natural course of the disease. However, such strategy should be definitely proven based on prospective clinical trial.

\section{Conflict of interest}

Authors indicate no potential conflicts of interests.

\section{Open Access}

This article is distributed under the terms of the Creative Commons Attribution Noncommercial License which permits any noncommercial use, distribution, and reproduction in any medium, provided the original author(s) and the source are credited.

\section{References}

1. Francken AB, Hoekstra HJ. Follow-up of melanoma patients: the need for evidence-based protocols. Ann Surg Oncol. 2009;16:804-5.

2. Fields RC, Coit DG. Evidence-based follow-up for the patient with melanoma. Surg Oncol Clin N Am. 2011;20:181-200.

3. Scally CP, Wong SL. Intensity of follow-up after melanoma surgery. Ann Surg Oncol. 2014;21:752-7.

4. Weiss M, Loprinzi CL, Creagan ET, Dalton RJ, Novotny P, O'Fallon JR. Utility of follow-up tests for detecting recurrent disease in patients with malignant melanomas. JAMA. 1995;274:1703-5.

5. Dummer R, Hauschild A, Guggenheim M, Keilholz U, Pentheroudakis G. Cutaneous melanoma: ESMO clinical practice guidelines for diagnosis, treatment and follow-up. Ann Oncol. 2012;23:vii86-91.

6. NCCN Clinical Practice Guidelines in Oncology. Melanoma v.2. 2014.

7. Garbe C, Hauschild A, Volkenandt M, et al. Evidence and interdisciplinary consensus-based German guidelines: diagnosis and surveillance of melanoma. Melanoma Res. 2007;17:393e9.

8. Johnson TM, Bradford CR, Gruber SB, Sondak VK, Schwartz JL. Staging workup, sentinel node biopsy, and follow-up tests for melanoma: update of current concepts. Arch Dermatol. 2004;140:107-13.

9. Rutkowski P, Wysocki PJ, Nowecki ZI, i wsp. Cutaneous melanoma-diagnostic and therapeutic guidelines in 2013. Onkologia w Praktyce Klinicznej. 2012;8:178-92.

10. Christianson DF, Anderson CM. Close monitoring and lifetime follow-up is optimal for patients with a history of melanoma. Semin Oncol. 2003;30:369-74.

11. Francken $A B$, Bastiaannet E, Hoekstra HJ. Follow-up in patients with localized primary cutaneous melanoma. Lancet Oncol. 2005;6:608-21.

12. Francken AB, Shaw HM, Accortt NA, Soong SJ, Hoekstra HJ, Thompson JF. Detection of first relapse in cutaneous melanoma patients: implications for the formulation of evidence-based follow-up guidelines. Ann Surg Oncol. 2007;14:1924-33.

13. Francken AB, Accortt NA, Shaw HM, et al. Follow-up schedules after treatment for malignant melanoma. $\mathrm{Br} \mathrm{J}$ Surg. 2008;95:1401-7.

14. Garbe C, Paul A, Kohler-Spath H, et al. Prospective evaluation of a follow-up schedule in cutaneous melanoma patients: recommendations for an effective follow-up strategy. J Clin Oncol. 2003;21:520-9.

15. Meyers MO, Yeh JJ, Frank J, et al. Method of detection of initial recurrence of stage II/III cutaneous melanoma: analysis of utility of follow-up staging. Ann Surg Oncol. 2009;16:941-7. 
16. Romano E, Scordo M, Dusza SW, Coit DG, Chapman PB. Site and timing of first relapse in stage III melanoma patients: implications for follow-up guidelines. J Clin Oncol. 2010;28:3042-7.

17. Pflugfelder A, Kochs C, Blum A, et al. Malignant melanoma S3-guidline "Diagnosis, therapy and follow-up of melanoma". J Dtsch Dermatol Ges. 2013;Suppl 6:1-126.

18. van der Leest RJ, van Steenbergen LN, Hollestein LM, de Vries E, Nijsten T, van Akkooi AC, Janssen-Heijnen ML, Coebergh JW. Conditional survival of malignant melanoma in The Netherlands: 1994-2008. Eur J Cancer. 2014;50:602-10.

19. Rueth NM, Xing Y, Chiang YJ, Cromwell KD, Ross MI, Lee JE, Gershenwald JE, Royal RE, Cormier JN. Is surveillance imaging effective for detecting surgically treatable recurrences in patients with melanoma? A comparative analysis of stage-specific surveillance strategies. Ann Surg. 2014;259:1215-22.

20. Turner RM, Bell KJ, Morton RL, et al. Optimizing the frequency of follow-up visits for patients treated for localized primary cutaneous melanoma. J Clin Oncol. 2011;29(35):4641-6.

21. Autier P, Coebergh JW, Boniol M, Dore JF, de Vries E, Eggermont AM. Management of melanoma patients: benefit of intense follow-up schedule is not demonstrated. J Clin Oncol. 2003;21:3707.

22. Rychetnik L, McCaffery K, Morton RL, Thompson JF, Menzies SW, Irwig L. Follow-up of early stage melanoma: specialist clinician perspectives on the functions of follow-up and implications for extending follow-up intervals. J Surg Oncol. 2013;107(5):463-8.

23. Einwachter-Thompson J, MacKie RM. An evidence base for reconsidering current follow-up guidelines for patients with cutaneous melanoma less than $0.5 \mathrm{~mm}$ thick at diagnosis. Br J Dermatol. 2008;159:337e41.
24. Balfounta ML, Beauchet A, Chagnon S, et al. Ultrasonography or palpation for detection of melanoma nodal invasion: a meta-analysis. Lancet Oncol. 2004;5:673e80.

25. Voit C, Mayer T, Kron M, et al. Efficacy of ultrasound B-scan compared with physical examination in follow-up of melanoma patients. Cancer. 2001;91:2409-16.

26. Kruger U, Kretschmer L, Thoms KM, Padeken M, Peter Bertsch H, Schon MP, Zutt M. Lymph node ultrasound during melanoma follow-up significantly improves metastasis detection compared to clinical examination alone: a study on 433 patients. Melanoma Res. 2011;21:457-63.

27. Morton DL, Thompson JF, Ochran AJ, et al. Sentinelnode biopsy or nodal observation in melanoma. NEJM. 2006;355:1307-17.

28. Leiter U, Buettner PG, Bohnenberger K, Eigentler T, Meier F, Moehrle M, Breuninger H, Garbe C. Sentinel lymph node dissection in primary melanoma reduces subsequent regional lymph node metastasis as well as distant metastasis after nodal involvement. Ann Surg Oncol. 2010;17:129-37.

29. Bamboat ZM, Konstantinidis IT, Kuk D, Ariyan CE, Brady MS, Coit DG. Observation after a positive sentinel lymph node biopsy in patients with melanoma. Ann Surg Oncol. 2014; May 16 [Epub ahead of print].

30. Morton RL, Craig JC, Thompson JF. The role of surveillance chest $\mathrm{x}$-rays in the follow-up of high-risk melanoma patients. Ann Surg Oncol. 2009;16:571-7.

31. Tsao H, Feldman M, Fullerton JE, Sober AJ, Rosenthal D, Goggins W. Early detection of asymptomatic pulmonary melanoma metastases by routine chest radiographs is not associated with improved survival. Arch Dermatol. 2004;140:67-70.

32. Forschner A, Eigentler TK, Pflugfelder A, Leiter U, Weide B, Held L, Meier F, Garbe C. Melanoma staging: facts and controversies. Clin Dermatol. 2010;28:275-80. 